\section{Kinder opiatabhängiger Mütter - Ein Überblick}

Ekkehart Englert ${ }^{1}$, Markus Ziegler ${ }^{2}$

${ }^{1}$ Klinik für Kinder- und Jugendpsychiatrie und Psychotherapie HELIOS Klinikum Erfurt

2 Brent, Kensington \& Chelsea and Westminster Substance Misuse Service, London SW10 9NG
Zusammenfassung: Kinder von opiatabhängigen Müttern stellen eine besondere Hochrisikogruppe dar. Kinder, die nach der Entbindung zu einem großen Prozentsatz einen Opiatentzug durchmachen, treffen auf eine durch eigene traumatisierende Erfahrungen, Abhängigkeitserkrankung und schwere psychische Probleme in ihrer Erziehungskompetenz erheblich eingeschränkte Mutter, die meist unter extrem ungünstigen psychosozialen Bedingungen lebt.

Die in der vorliegenden Literaturübersicht zusammengefassten Längsschnittuntersuchungen beschreiben übereinstimmend in den ersten Lebensjahren der Kinder opiatabhängiger Mütter Wachstumsretardierungen sowie Wahrnehmungs- und Interaktionsstörungen. Die intellektuelle Entwicklung scheint nicht beeinträchtigt, in manchen Untersuchungen finden sich jedoch Hinweise auf Sprachentwicklungsverzögerungen. Im Schulalter zeigen sich in allen Studien erhöhte Aggressivität und Impulsivität, Störungen im Sozialverhalten, Schulprobleme, Delinquenz und früher Alkohol- und Drogenkonsum.

Eine umfassende interdisziplinäre Betreuung der Frauen ab der Schwangerschaft einschließlich Substitution, Beratung, Anleitung und aufsuchenden Hilfen ist unbedingt erforderlich und kann die Gesamtprognose entscheidend verbessern.

Schlüsselwörter: Kinder - Opiatababhängige Mütter

Children of Opiate-Addicted Mothers - a Review: Children of opiate-addicts constitute a special high-risk group. Most of these children (between 40 and 95 pct.) develop within the first weeks pp. a so-called „neonatal abstinence syndrome" (NAS) which shows a well documented dosageeffect-relationship. First descriptions of NAS in children of morphine-addicted mothers were reported from Berlin in 1930. In reviewing the past three decades of literature on the development of children of opiate-addicted mothers the hypothesis can be formulated, that a mixture of neonatal abstinence effects (with irritability, heightened arousal, and distractibility) with their postnatal environmental-interactive sequelae and a lack of familial coping resources may lead to the development of hypercinetic-attention deficit-syndromes and conduct disorders at school age resulting in serious school problems.

Suchttherapie 2001; 2: 143-151

(c) Georg Thieme Verlag Stuttgart · New York

ISSN 1439-9903
Studies on children of drug-addicts at school age agree in the following trends: In standardised intelligence tests children of drug-addicts show no significant difference to controls in total-IQ but lower verbal IQ's and to a far greater extent conduct disorders and school-problems. They show more impulsivity, aggression and attention-problems as well as a variety of other externalising behaviour problems as indicated by CBCL scores within the clinical range.

Methadone treatment may be an important factor in stabilising the psycho-social situation of pregnant addicts. Results from our study at Frankfurt University Hospital show clearly, that methadone-maintained mothers lived in more stable socio-economic conditions than heroin-addicted women, and they cared significantly better for their babies. Reports from different experimental interventions or homebased intervention programs with families of substance abusers indicate positive changes in parenting skills, parental drug use, and family management.

Key words: Children - Opiat-addicted Mothers

\section{Einführung}

Unter den Kindern von abhängigkeitserkrankten Eltern nehmen Kinder opiatabhängiger Mütter eine Sonderstellung ein. Dies betrifft die spezifische psychosoziale Lebenssituation der Mütter, die mit dem intravenösen Drogenkonsum verbundenen gesundheitlichen Risiken und die Tatsache, dass das Leben des Kindes bereits intrauterin mit einem unfreiwilligen Opiatkonsum und körperlicher Abhängigkeitsentwicklung beginnt [1].

Der alleinige Konsum von Opiaten stellt sowohl in der nationalen als auch internationalen Drogenszene eher die Ausnahme denn die Regel dar. In einer deutschen epidemiologischen Untersuchung fand sich bei den Personen mit Opiatmissbrauch/-abhängigkeit am häufigsten ein gleichzeitiger Missbrauch von Kokain in 66\%, gefolgt von Cannabis und Amphetaminen [2]. Daher sind, wenn nicht speziell erwähnt, auch mehrfach abhängige Mütter und ihre Kinder in die folgende Literaturübersicht mit einbezogen, sofern Opiate die Hauptdroge darstellen.

\section{Historischer Exkurs}

„Eine Frau, die seit mehreren Jahren täglich 10-20 cg Morphium brauchte, wurde schwanger. Unmittelbar nach der Geburt fing das Kind zu schreien an und war dauernd, Tag 
und Nacht, sehr unruhig und verstört. Es trank begierig die Flasche, brach aber immer wieder alles aus. Im Verlauf von etwa 60 Stunden war das Kind sehr elend. Nach Verabreichung von 2 mal tägl. $1 / 2$ Tropfen Tinct. Thebaica hörte das Erbrechen auf. Das Kind war ruhig, fing an zu schlafen und hat sich später normal entwickelt.“

Diese Beschreibung ist der Zeitschrift „Medizinische Klinik“ aus dem Jahre 1930 entnommen, in welcher Langstein aus Berlin [3] mehrere Fälle von Kindern morphiumsüchtiger Frauen („Morphinistinnen“) schilderte, die post partum hochgradige Unruhe und Krampfanfälle, anhaltendes Erbrechen und Dystrophie gezeigt hatten mit Besserung der Symptomatik unter Gabe einer opiumhaltigen Tinktur. In seinem Beitrag in demselben Jahrgang dieser Zeitschrift interpretierte Weiss [4] die vorgenannten Schilderungen dergestalt, „dass bei Neugeborenen morphiumsüchtiger Frauen richtige und hochgradige Abstinenzerscheinungen auftreten können, die nach den für Erwachsene geltenden Regeln zu behandeln sind“. $\mathrm{Zu}$ Beginn des 19. Jahrhunderts war Opium ein weit verbreitetes Heilmittel. In der Frauenheilkunde und Geburtshilfe spielte es eine große Rolle und gerade in den unteren sozialen Schichten war es gang und gäbe, Neugeborene und Kleinkinder mit Opiumsäften zu beruhigen [5].

\section{Zum Konstrukt der „Risikofaktoren“}

Kinder opiatabhängiger Mütter sind während ihrer prä- und postnatalen Entwicklung potenziell einer Reihe von Risiken ausgesetzt [6], die sich entweder direkt aus dem Drogenkonsum der Mutter ableiten oder indirekt verursacht sind. Groeneweg et al. [7] unterscheiden hierbei primäre von sekundären Risikofaktoren: Zu den primären Risiken zählen die transplazentare Drogenexposition, Mangelernährung und eine hohe Prävalenz von Infektionskrankheiten (Hepatitis, Geschlechtskrankheiten, HIV) bei der Mutter. Als sekundäre Risikofaktoren kommen die besonders ungünstigen bzw. drogenmilieuspezifischen psychosozialen Bedingungen hinzu. Viele dieser Risiken sind bis heute nur unzureichend erforscht. Teratogene und toxikologische Schäden konnten, im Gegensatz zu Alkohol, bei reinem Opiatkonsum bis jetzt nicht festgestellt werden [8-10].

\section{Empirische Befunde}

\section{Epidemiologie}

Bislang liegen in der internationalen Literatur keine epidemiologisch gesicherten Angaben zur Prävalenz von Kindern opiatabhängiger Mütter vor, daher sind wir auf grobe Schätzungen angewiesen. Das Bundesministerium für Gesundheit [11] gab 1997 die Zahlen der Konsumenten so genannter „harter“ Drogen (Opiate, Kokain, Amphetamine), die mit hoher Intensität konsumieren, mit 100000-150000 an. 1994 wurde die Anzahl der Kinder opiatabhängiger Eltern in Deutschland auf 30000 geschätzt [12].

Bei Untersuchungen von Kleiber und Pant [13] in der Berliner Drogenszene waren ca. $30 \%$ aller i.v. Opiatabhängigen weiblichen Geschlechts, davon $80 \%$ im gebärfähigen Alter. Ein Drittel dieser Frauen hatte bereits ein Kind geboren, ein Viertel der männlichen Opiatabhängigen waren Väter. Relativ aktuelle Daten aus ambulanten Beratungs- und Behandlungs- stellen für Suchtkranke in Deutschland, der EBIS-Jahresstatistik 1999, [14] zeigen, dass von den Klienten mit der Hauptdiagnose „Opiate“ 31\% der Männer und 45\% der Frauen mindestens ein Kind hatten.

Eine Schweizer Screeninguntersuchung [15] fand im Mekonium von Neugeborenen in 7\% einen Nachweis für illegale Drogen. Wolstein u. Mitarb. berichten aus Essen, dass an der dortigen Universitätsfrauenklinik 2,2\% der entbundenen Frauen nach eigenen Angaben opiatabhängig waren [16]; in der psychiatrischen Ambulanz der Rheinischen Kliniken hatten 56\% der wegen Drogenabhängigkeit behandelten Frauen eigene Kinder [16].

\section{Beeinflussung der Mutterrolle durch Psychopathologie und Suchtverhalten}

Nach Davis [17] sind vier Aspekte charakteristisch für substanzabhängige Mütter: Kindheitstraumata, psychische Störungen, niedriger sozioökonomischer Status und ambivalente Auseinandersetzung mit der Mutterrolle.

\section{Kindheitstraumata}

In ihrem eigenen Elternhaus hatten die Frauen oft kein Modell für adäquate elterliche Fürsorge, eine stabile Familie hat oft nicht existiert [17]. In einer qualitativen InterviewUntersuchung fand Coyer [18] bei kokainabhängigen Müttern geringes Selbstwertgefühl, Schwierigkeiten, eine eigene mütterliche Identität $\mathrm{zu}$ entwickeln, Isolation von Freunden und Familie und chronische Stressbelastung. Die Mehrzahl der Frauen hat wiederholt Erfahrungen von Trauma und Verlust durchgemacht, was Gefühle von Depression, Angst, Minderwertigkeit und unkontrollierter Panik zur Folge hat [19]. Im Einzelnen tauchen immer wieder Tod der Eltern, Scheidung, Gewalt in der Familie, körperlicher und sexueller Missbrauch [20], Deprivation und Heimaufenthalte in der Lebensgeschichte von Substanzabhängigen auf [13,21-24] mit nachweislichen Auswirkungen auf die Erziehungskompetenz und häufigeren Fremdunterbringungen der Kinder [24].

\section{Psychiatrische Komorbidität}

Bei drogenabhängigen Müttern ist mit einer hohen Prävalenz von weiteren psychischen Störungen zu rechnen: In einer Übersichtsarbeit nennt Salloum [25] Raten für psychiatrische Komorbidität von $53,1 \%$. Eine rund doppelt so hohe psychiatrische Symptombelastung der weiblichen im Vergleich zu den männlichen Klienten in verschiedenen Drogen-Therapieeinrichtungen in Großbritannien fanden Marsden et al. [26]: Angstsymptomatik in 32,3 vs. $17,5 \%$, Depressivität in 29,7 vs. $14,9 \%$.

Bei Methadonsubstituierten in Basel betrug die psychiatrische Komorbidität 75,2\%, am häufigsten Persönlichkeitsstörungen, gefolgt von depressiven und schizophrenen Störungen [27]. Bei Patienten, die sich zur Entgiftung in der psychiatrischen Universitätsklinik Tübingen befanden, fand sich eine Lebenszeitprävalenz psychischer Störungen von 55\%, dabei vorherrschend Angst- und Zwangsstörungen und affektive Störungen [28]. In verschiedenen Studien [21,17,29,30] werden die Frauen als sozial isoliert, depressiv, unsicher, ohne Selbstwertgefühl und mit Schuldgefühlen behaftet beschrieben. 


\section{Beobachtungen zum Erziehungsverhalten}

$\mathrm{Zu}$ dieser gerade angeführten mütterlichen Problematik kommt hinzu, dass das Kind durch entzugsbedingtes untypisches Verhalten den hohen Erwartungen der Mutter oft nicht gerecht wird [31], was zur Belastung der Mutter-Kind-Beziehung führt. Bernstein et al. [32] beobachteten, dass methadonsubstituierte Mütter weniger häufig auf die Interaktionswünsche ihrer Kinder eingingen und auch weniger häufig zur Kommunikation anregten. Van Baar [33] sah bei den abhängigen Müttern weniger Einfühlungsvermögen und stellte fest, dass Mütter in der drogenfreien Kontrollgruppe mehr und angemessener mit ihren Kindern spielten. Auch vermehrt aversives Verhalten der Mütter ihren 2- bis 6-jährigen Kindern gegenüber in Form von Provokationen, Drohungen und Befehlen wurde beobachtet [34].

\section{Psychosoziale Bedingungen}

Opiatabhängige Mütter gehören überwiegend unteren sozialen Schichten an; typischerweise sind die Mütter allein stehend, ohne stabile Partnerbeziehung und die Wohnverhältnisse sind oft unzureichend. 15-24\% der Drogenkonsumenten sind wohnungslos [13]. Der Bildungsstand ist niedrig, der Lebensunterhalt wird überwiegend durch staatliche Unterstützungsgelder, Beschaffungskriminalität und Prostitution finanziert. 50-70\% der Abhängigen in dem untersuchten Kollektiv hatten Hafterfahrungen. Bei fortgesetzter Polytoxikomanie wird der überwiegende Teil der verfügbaren zeitlichen und finanziellen Mittel für die Drogenbeschaffung/Beschaffungskriminalität eingesetzt und steht dann nicht mehr für die Versorgung des Kindes zur Verfügung [35]; je länger die Suchterkrankung besteht, desto größer wird die Verschuldung; fast die Hälfte der „Langzeitfixer“ (>10 Jahre) hat Schulden von mehr als DM 10000 [13].

Die Partnerbeziehungen drogenabhängiger Frauen sind instabil, nur jeder zehnte Drogenkonsument war in der o.g. Untersuchung verheiratet, aber fast ein Drittel der Frauen hatte mindestens ein Kind. Dabei sind weibliche Drogenabhängige häufiger mit gleichfalls drogenabhängigen Partnern zusammen als männliche [36], was gleichzeitig die biologischen und die psychosozialen Risiken für die Kinder dieser Mütter vergrößert.

\section{Die Schwangerschaft}

Für viele der Frauen ist die Schwangerschaft ein unerwartetes und ungeplantes Ereignis [21]. Nur 6\% der an der Ambulanz der Rheinischen Kliniken in Essen befragten Drogenabhängigen gaben an, regelmäßig Verhütungsmittel zu verwenden; eine Schwangerschaft wird aufgrund der unter fortgesetztem Opiatkonsum häufigen Amenorrhö bzw. Zyklusunregelmäßigkeiten erst spät bemerkt [16]. Die Mehrzahl der Frauen setzt den Opiatkonsum in der Schwangerschaft und auch nach der Geburt fort $[37,38]$.

Die Gefühle dem Kind gegenüber sind oft ambivalent; auf der einen Seite wird die Geburt des Kindes mit Hoffnung auf Ausstieg aus dem Drogenmilieu verbunden, auf der anderen Seite kann jedoch das Kind auch dazu dienen, eine gewisse innere Leere auszufüllen und damit zu einem Substitut für eigene unerfüllte Wünsche werden [21]. Aus dem Konflikt des Wissens um die Drogenexposition des ungeborenen Kindes und die eigene Unfähigkeit, den Drogenkonsum zu beenden, resultieren Schuld- und Versagensgefühle [17].

Die meisten substanzabhängigen Frauen vernachlässigen ihre Gesundheit und bemühen sich kaum um eine adäquate Schwangerschaftsvorsorge [39-41].

Die meisten Autoren beschreiben eine erhöhte Rate an Schwangerschafts- und Geburtskomplikationen [42,43]. Hervorzuheben ist das zunehmende Problem der HIV-Infektion. Kleiber und Pant [13] geben die HIV-Antikörperprävalenz in der Gruppe der Drogenabhängigen mit $12-30 \%$ an, Lejeune et al. [41] fanden bei 59 polytoxikomanen Müttern 21\% HIVpositive. In unseren eigenen Erhebungen [44] an der neonatologischen Abteilung des Universitätsklinikums Frankfurt war der Anteil der HIV-positiven unter den opiatabhängigen Müttern von 10\% im Jahre 1988 auf 60\% im Jahre 1995 angestiegen. In der Folge besteht die Gefahr der Weitergabe des Virus an das Kind, entweder transplazentar oder postnatal durch Stillen. Diese sog. vertikale Transmissionsrate wird mit 20-40\% angegeben [45,46].

\section{Die kindliche Entwicklung bis zum Alter von 3 Jahren}

Neonatales Abstinenzsyndrom (NAS)

Konsumiert die Mutter während der Schwangerschaft Opiate - in Form des fortgesetzten Abusus oder im Rahmen einer Substitution - tritt innerhalb der ersten 4 bis 6 Wochen post partum bei der Mehrheit der Kinder (zwischen 50 und 95\% $[47,48]$, siehe Tab. 2 ein sog. „Neonatales Abstinenzsyndrom“ (NAS) auf, das eine eindeutige Dosis-Wirkungs-Beziehung aufweist: Die Höhe der während der Schwangerschaft konsumierten Opiatdosis ist entscheidend für das Auftreten bzw. den Schweregrad der Entzugssymptomatik bei dem Neugeborenen $[48,49]$. Loretta Finnegan [50] entwickelte eine Skala zur Einschätzung des NAS, in der die 21 häufigsten Symptome erfasst werden. Dazu gehören exzessives, hochfrequentes Schreien, übersteigerte Reflexe, Tremor, gesteigerter Muskeltonus, Anfälle, Schwitzen und weitere respiratorische und gastrointestinale Störungen; therapeutisch wird die Gabe von Phenobarbital empfohlen, womit in abgestufter Dosierung der Entzug erfolgt.

Der Entzug beginnt meist innerhalb der ersten Lebenstage (im Durchschnitt am 2. Tag, am häufigsten am 3. Tag pp. [51]), in einem Zeitraum von 6 Tagen bis zu 8 Wochen können noch Symptome beobachtet werden. Selbst nach medikamentöser Behandlung kann Übererregbarkeit noch bis zu 3 Monaten nach Entlassung vorkommen. Eine vergleichbare Entzugssymptomatik lässt sich auch tierexperimentell bei neugeborenen Ratten erzeugen, deren Mütter in den letzten zwei Wochen der Gestation Methadon erhalten hatten [52]. Der Methadonentzug ist, aufgrund der weitaus längeren Halbwertszeit der Substanz, schwerer und langwieriger als der reine Heroinentzug $[53,54]$.

In der Literatur wird das Auftreten des neonatalen Abstinenzsyndroms mit 20-90\% angegeben, bei einem Mittelwert von ca. 70\%. Tab. 1 gibt eine Übersicht über die häufigsten Symptome des NAS [55]. Die zentralnervösen Störungen (außer Krampfanfälle) stehen mit 75-100\% an der Spitze der 
Häufigkeitsverteilung. Die Angaben zur Häufigkeit zerebraler Krampfanfälle sind, wie aus Tab. 2 ersichtlich, sehr unterschiedlich; es werden zwischen 3 und $65 \%$ entzugsbedingter Anfälle beschrieben.

Tab. 1 Häufigkeit der einzelnen Entzugssymptome bei 138 Neugeborenen mit NAS (nach Finnegan \& Kandall [55])

\begin{tabular}{lc}
\hline Symptom & rel. Häufigkeit \\
\hline Tremor & \\
schrilles Schreien & $96 \%$ \\
Schnupfen & $95 \%$ \\
erhöhter Muskeltonus & $83 \%$ \\
Erbrechen & $82 \%$ \\
Atemfrequenz $>60 /$ min & $74 \%$ \\
Fütterstörung & $66 \%$ \\
hyperaktiver Mororeflex & $65 \%$ \\
anhaltendes schrilles Schreien & $62 \%$ \\
dünner Stuhl & $54 \%$ \\
Schwitzen & $51 \%$ \\
Fieber $<38,2{ }^{\circ} \mathrm{C}$ & $49 \%$ \\
wässriger Stuhl & $29 \%$ \\
Fieber $>38,2{ }^{\circ} \mathrm{C}$ & $12 \%$ \\
generalisierte Krampfanfälle & $3 \%$ \\
Dehydratation & $1 \%$ \\
\hline \hline
\end{tabular}

Wachstum/Reife

Einen Überblick über die Parameter Geburtsgewicht und Gestation bietet Tab. 2.

Übereinstimmend kann festgestellt werden, dass das mittlere Geburtsgewicht niedriger ausfällt. Der Anteil der Neugeborenen mit niedrigem Geburtsgewicht liegt ungefähr zwischen 20 und 40\%. Ebenso erhöht ist der „Small-for-date“-Anteil.
Nach Abklingen der Entzugserscheinungen scheinen sich die Neugeborenen weitgehend normal zu entwickeln. Trotzdem wirkt das gestörte Verhalten auf die Mutter-Kind-Interaktion ein und stellt potenziell einen prädisponierenden Faktor für spätere Psychopathologie dar [56].

Eine Gesamtübersicht der Literatur zu Längsschnittuntersuchungen von Kindern drogenabhängiger Mütter, überwiegend aus den USA und Skandinavien, findet sich in Tab. 3. Die Forscher kommen zu unterschiedlichen Schlüssen. Manche Autoren [57] konnten bei ihrer Untersuchung von Kindern bis zu zwei Jahren keine signifikanten Beeinträchtigungen der kognitiven Leistungen feststellen und berichteten von einer weitgehenden Normalisierung der anfangs gefundenen Auffälligkeiten [58]. Lifschitz et al. [59] hingegen konnten keine Normalisierung feststellen. In ihrem Kollektiv von Kindern bis zu 4 Jahren blieben die intellektuellen Leistungen signifikant unter den Werten der Kontrollgruppe. Diese Ergebnisse bestätigte auch van Baar [33], die insbesondere eine verzögerte Sprachentwicklung beschreibt.

Weitgehend Übereinstimmung herrscht bei den Forschern darüber, dass die Familiensituation, in der das Kind aufwächst, mehr Aussagekraft über die weitere Entwicklung des Kindes besitzt als das Ausmaß des Opiatkonsums der Mutter während der Schwangerschaft [59].

\section{Vorschulalter und Schulalter (3-16 Jahre)}

Auch hier kommen Forscher zu unterschiedlichen, aber in der Tendenz konvergierenden Ergebnissen. In einer Untersuchung von van Baar et al. [60] über Kinder bis zum Alter von 5,5 Jahren ließen sich bei der motorischen Entwicklung keine Unterschiede zur Kontrollgruppe feststellen. Sehr wohl konnten jedoch vermehrt Verhaltensauffälligkeiten beobachtet werden, wie z.B. gesteigerte Aggressivität, depressive

Tab. 2 Häufigkeit postpartaler Komplikationen bei Kindern von Opiatabhängigen

\begin{tabular}{|c|c|c|c|c|c|c|c|c|c|}
\hline & Jahr & Ref. & $\mathrm{N}$ & NAS & $\begin{array}{l}\text { Krampf- } \\
\text { anfälle }\end{array}$ & $\begin{array}{l}\text { Frühgeb. } \\
\text { vor } 34 . \text { SSW }\end{array}$ & $\begin{array}{l}\text { Geb.-Gew. } \\
<2500 \mathrm{~g}\end{array}$ & $\begin{array}{l}\text { „small } \\
\text { for date“ }\end{array}$ & $\begin{array}{l}\text { perinat. } \\
\text { Kompli- } \\
\text { kationen }\end{array}$ \\
\hline $\begin{array}{l}\text { Stimmel \& Adamsons, } \\
\text { New York }\end{array}$ & 1976 & [53] & & & & & & & \\
\hline $\begin{array}{ll}\text { Methadon } \\
\text { Heroin }\end{array}$ & & & $\begin{array}{l}31 \\
57\end{array}$ & $\begin{array}{l}58 \% \\
51 \%\end{array}$ & & & $\begin{array}{l}23 \% \\
26 \%\end{array}$ & & $\begin{array}{l}16 \% \\
40 \%\end{array}$ \\
\hline Sardemann et al., Kopenhagen & 1976 & {$[61]$} & 19 & $84 \%$ & & $25 \%$ & $37 \%$ & & \\
\hline Olofsson et al., Kopenhagen & 1983 & {$[10]$} & 89 & $85 \%$ & $12 \%$ & & & & \\
\hline $\begin{array}{cl}\text { Lifschitz, Houston } & \begin{array}{l}\text { Methadon } \\
\text { Heroin }\end{array}\end{array}$ & 1985 & [59] & $\begin{array}{l}26 \\
25\end{array}$ & $\begin{array}{l}88 \% \\
68 \%\end{array}$ & & & & & \\
\hline Klenka, GB & 1986 & [94] & 25 & $76 \%$ & & & & $32 \%$ & \\
\hline van Baar, Amsterdam & 1989 & [95] & 35 & $94 \%$ & $3 \%$ & $26 \%$ & & $12 \%$ & \\
\hline Little et al., Dallas & 1991 & [8] & 47 & $43 \%$ & & & & & \\
\hline $\begin{array}{l}\text { Vering, Seeger et al., } \\
\text { Frankfurt/M. }\end{array}$ & 1992 & [49] & 20 & $95 \%$ & $65 \%$ & $20 \%$ & $45 \%$ & $15 \%$ & \\
\hline Bode et al., Basel & 1992 & {$[48]$} & 21 & $95 \%$ & $29 \%$ & $29 \%$ & & $38 \%$ & \\
\hline Schneider et al., Wien & 1996 & [77] & 17 & $87 \%$ & & $18 \%$ & $30 \%$ & & \\
\hline Agarwal et al., Singapur & 1999 & [51] & 38 & $47 \%$ & $2,6 \%$ & $5,3 \%$ & & $42 \%$ & \\
\hline Kelly et al., Melbourne & 2000 & {$[38]$} & 96 & $30 \%$ & & & & & \\
\hline
\end{tabular}


Tab. 3 Literaturübersicht zur Entwicklung von Kindern opiatabhängiger Mütter

\begin{tabular}{|c|c|c|c|c|c|c|c|}
\hline Untersuchung & Jahr & Drogen & $\mathrm{N}$ & Alter & Motorik & Intelligenz & Psychopathologie \\
\hline Fanshel & $\begin{array}{l}1975 \\
{[96]}\end{array}$ & verschiedene & 44 & & k. A. & $\varnothing$ Differenz & $\begin{array}{l}\text { Leistungs- und Verhaltens- } \\
\text { probleme in der Schule }\end{array}$ \\
\hline $\begin{array}{l}\text { Sardemann et al., } \\
\text { Kopenhagen }\end{array}$ & $\begin{array}{l}1976 \\
{[61]}\end{array}$ & Heroin/Methad. & 19 & bis 2,8 & $\begin{array}{l}\varnothing \text { Auf- } \\
\text { fälligkt. }\end{array}$ & $\begin{array}{l}\text { durchschnittl., aber } \\
\text { Sprachentwicklungs- } \\
\text { verzögerungen }\end{array}$ & k. A. \\
\hline Wilson et al., Houston & $\begin{array}{l}1979 \\
{[62]}\end{array}$ & Heroin/Methad. & 22 & $\begin{array}{l}3-6 \\
\varnothing 4,5\end{array}$ & k. A. & $\begin{array}{l}\varnothing \text { ges. IQ, Sprache } \downarrow \\
\text { Wahrnehmung } \downarrow,\end{array}$ & $\begin{array}{l}\text { Impulsivität, Aggressivität, } \\
\text { Störungen des Sozialverhaltens }\end{array}$ \\
\hline Strauss et al. & $\begin{array}{l}1979 \\
{[97]}\end{array}$ & Methadon & 33 & 5 & $\begin{array}{l}\text { feinmotor. } \\
\text { Koordina- } \\
\text { tion } \downarrow\end{array}$ & $\varnothing$ Differenz & $\begin{array}{l}\text { hypermotorisches Verhalten } \\
\text { „ungesteuerte Aktivität“ }\end{array}$ \\
\hline \multirow[t]{2}{*}{ Sowder \& Burt } & $\begin{array}{l}1980 \\
{[40]}\end{array}$ & Heroin/Methad. & 34 & $3-7$ & $\begin{array}{l}42 \% \text { neuro- } \\
\text { log./motor. } \\
\text { auffällig }\end{array}$ & ges. IQ $\downarrow$ & $\begin{array}{l}\text { ängstlicher, unsicherer, kürzere } \\
\text { Aufmerksamkeitsspanne }\end{array}$ \\
\hline & & & & $8-17$ & k. A. & k. A. & $\begin{array}{l}\text { Schulschwänzen, Leistungs-/ } \\
\text { Verhaltensprobleme, Delinquenz, } \\
\text { Substanzmissbrauch }\end{array}$ \\
\hline $\begin{array}{l}\text { Olofsson et al., } \\
\text { Kopenhagen }\end{array}$ & $\begin{array}{l}1983 \\
{[10]}\end{array}$ & Methadon & 72 & $\begin{array}{l}1-10 \\
\varnothing 3,5\end{array}$ & $\begin{array}{l}21 \% \\
\text { psychom. } \\
\text { Entwicklungs- } \\
\text { störungen }\end{array}$ & k. A. & $\begin{array}{l}\text { in } 56 \% \text { Aufmerksamkeitsstörun- } \\
\text { gen, Hyperaktivität, Aggressivität, } \\
\text { fehlende soziale Hemmungen }\end{array}$ \\
\hline Kaltenbach \& Finnegan & $\begin{array}{l}1984 \\
{[57]}\end{array}$ & Methadon & 35 & 4 & $\varnothing$ Differenz & $\varnothing$ Differenz & k. A. \\
\hline $\begin{array}{l}\text { Lifschitz et al., } \\
\text { Houston }\end{array}$ & $\begin{array}{l}1985 \\
{[59]}\end{array}$ & Heroin/Methad. & 30 & 3 & $\varnothing$ Differenz & $\varnothing$ Differenz & k. A. \\
\hline Bauman \& Levine & $\begin{array}{l}1986 \\
{[98]}\end{array}$ & Methadon & 70 & $3-6$ & $\begin{array}{l}\text { schlechtere } \\
\text { Werte }\end{array}$ & $\begin{array}{l}\text { ges. IQ } \downarrow \text {, expressive } \\
\text { Sprache } \downarrow\end{array}$ & k. A. \\
\hline $\begin{array}{l}\text { Groeneweg et al., } \\
\text { Rotterdam }\end{array}$ & $\begin{array}{l}1988 \\
{[7]}\end{array}$ & Heroin/Methad. & 66 & $2-7$ & $\begin{array}{l}\varnothing \text { Auf- } \\
\text { fälligkt. }\end{array}$ & $\varnothing$ (durchschnittlich) & $\begin{array}{l}\text { CBCL: bei den 6- bis 7-Jährigen } \\
\text { sig. } \downarrow \text { Soc. Competence und } \\
\text { sig. } \uparrow \text { Gesamtscore }\end{array}$ \\
\hline \multirow[t]{2}{*}{$\begin{array}{l}\text { van Baar, } \\
\text { Amsterdam }\end{array}$} & $\begin{array}{l}1990 \\
{[63]}\end{array}$ & Heroin/Methad. & 35 & $2 \frac{1}{2}$ & $\begin{array}{l}\varnothing \text { Auf- } \\
\text { fälligkt. }\end{array}$ & $\begin{array}{l}\text { nonverbal unauff. } \\
\text { Sprachentwicklungs- } \\
\text { verzögerung }\end{array}$ & $\varnothing$ Auffälligkeit \\
\hline & $\begin{array}{l}1991 \\
{[33]}\end{array}$ & Heroin/Methad. & 25 & $4 / 2$ & $\begin{array}{l}\varnothing \text { Auf- } \\
\text { fälligkt. }\end{array}$ & ges. IQ $\downarrow$ & $\begin{array}{l}\text { Aufmerksamkeitsstörungen, } \\
\text { unkooperat., } \\
\text { CBCL: sig. } \uparrow \text { Gesamtscore, sig. } \uparrow \\
\text { Aggression }\end{array}$ \\
\hline $\begin{array}{l}\text { de Cubas \& Field, } \\
\text { Miami }\end{array}$ & $\begin{array}{l}1993 \\
{[65]}\end{array}$ & Methadon & 20 & $\begin{array}{l}6-13 \\
\varnothing 8,5\end{array}$ & $\varnothing$ Differenz & $\varnothing$ Differenz & $\begin{array}{l}\text { CBCL: sig. } \uparrow \text { Gesamtscore, sowie } \\
\text { fast alle Subscores } \uparrow \text { (int./ext.), } \\
\uparrow \text { Aufmerksamkeits- und Schul- } \\
\text { schwierigkeiten }\end{array}$ \\
\hline $\begin{array}{l}\text { Griffith et al., } \\
\text { Chicago }\end{array}$ & $\begin{array}{l}1994 \\
{[66]}\end{array}$ & „Polydrug-user“ & 75 & 3 & $\begin{array}{l}\text { k. A., gerin- } \\
\text { gerer Kopf- } \\
\text { umfang }\end{array}$ & $\varnothing$ Differenzen & $\begin{array}{l}\text { CBCL: sig. } \uparrow \text { external. Score, } \\
\uparrow \text { Aggression, } \uparrow \text { Destruktivität }\end{array}$ \\
\hline $\begin{array}{l}\text { Wilens et al., } \\
\text { Boston }\end{array}$ & $\begin{array}{l}1995 \\
{[67]}\end{array}$ & Methadon & 44 & $\begin{array}{l}4-18 \\
\varnothing 10,4\end{array}$ & k. A. & k. A. & $\begin{array}{l}\text { CBCL: sig. } \downarrow \text { Soc. Competence und } \\
\text { sig. } \uparrow \text { Gesamtscore, v. a. Delin- } \\
\text { quenz } \uparrow, \text { Aufmerksamkeitsprobl. } \uparrow\end{array}$ \\
\hline $\begin{array}{l}\text { Merikangas et al., } \\
\text { New Haven }\end{array}$ & $\begin{array}{l}1998 \\
{[71]}\end{array}$ & $\begin{array}{l}\text { „Polydrug-user“ } \\
\text { inkl. Alkohol }\end{array}$ & 77 & $\begin{array}{l}7-17 \\
\varnothing 12,5\end{array}$ & k. A. & k. A. & $\begin{array}{l}\text { K-SADS: } 20 \% \text { Alkoholmissbr./ } \\
\text {-abhängigkt., } 25 \% \text { Nikotinabusus, } \\
10 \% \text { Drogenabusus }\end{array}$ \\
\hline $\begin{array}{l}\text { Koren et al., } \\
\text { Toronto }\end{array}$ & $\begin{array}{l}1998 \\
{[64]}\end{array}$ & $\begin{array}{l}\text { Kokain, } \\
\text { adoptiert }\end{array}$ & 23 & 2,8 & $\begin{array}{l}\varnothing \text { Diff. } \\
\text { geringerer } \\
\text { Kopfumfang }\end{array}$ & $\begin{array}{l}\text { ges. IQ } \varnothing \text { Differenz, } \\
\text { Verbal-IQ } \downarrow\end{array}$ & k. A. \\
\hline $\begin{array}{l}\text { Chasnoff et al., } \\
\text { Chicago }\end{array}$ & $\begin{array}{l}1998 \\
{[68]}\end{array}$ & $\begin{array}{l}\text { Kokain + } \\
\text { „Polydrug-user“ }\end{array}$ & 64 & $4-6$ & k. A. & $\varnothing$ sig. Differenzen & $\begin{array}{l}\text { CBCL: sig. } \uparrow \text { Gesamtscore, sowie } \\
\text { fast alle Subscores } \uparrow \text { (ext. > int.) }\end{array}$ \\
\hline $\begin{array}{l}\text { Nurco et al., } \\
\text { Baltimore }\end{array}$ & $\begin{array}{l}1999 \\
{[72]}\end{array}$ & $\begin{array}{l}\text { „Polydrug-user“ } \\
\text { in Substitution }\end{array}$ & 285 & $\begin{array}{l}12-17 \\
\varnothing 14,4\end{array}$ & k. A. & k. A. & $\begin{array}{l}64 \% \text { mit } 11 \text { Jahren bereits erste } \\
\text { Delikte, } 21 \% \text { Drogenabusus, } \\
\uparrow \text { Feindseligkeit }\end{array}$ \\
\hline
\end{tabular}


Verstimmungen und Probleme beim Umgang mit Gleichaltrigen und Erwachsenen. Ebenso gab es deutliche Defizite im kognitiven Bereich, vor allem bei der Sprachentwicklung. Mehrheitlich fanden die Untersuchungen keine Differenzen im Gesamt-IQ, jedoch zeigen mehrere Studien [61-64], dass die Kinder drogenabhängiger Mütter signifikant schlechtere Verbal-IQs aufwiesen als die jeweiligen Kontrollgruppen. Milieubedingte Gruppenunterschiede kann dabei allerdings nur die letztgenannte Studie von Koren et al. [64] weitgehend ausschließen, da die untersuchten Kinder nach der Geburt von Mittelschichtfamilien adoptiert worden waren.

Zur Einschätzung der Psychopathologie wurde in einem großen Teil der Follow-up-Untersuchungen [7,33,65-68] die Child Behavior Checklist (CBCL) von Achenbach [69] eingesetzt, ein weit verbreiteter standardisierter Elternfragebogen zu Verhaltens- und emotionalen Auffälligkeiten und somatischen Beschwerden von Kindern und Jugendlichen, der mittlerweile in mehr als 50 Sprachen übersetzt ist. Aus den Items werden u. a. zwei übergeordnete Skalen „internalisierende“ und „externalisierende“ Auffälligkeiten generiert. Wie Sie Tab. 3 entnehmen können, werden die Kinder drogenabhängiger Mütter in allen mit der CBCL durchgeführten Untersuchungen mit einem erhöhten Gesamtwert als auffällig eingeschätzt [7,33,65-68]; die einzelnen Problemskalen zeigen übereinstimmend erhöhte Aggressivität, Aufmerksamkeitsprobleme, Delinquenz bei erniedrigter sozialer Kompetenz.

Das Vorkommen gesteigerter Aggressivität konnte mehrfach bestätigt werden. Nur in einer Untersuchung [70] wurde bisher ein Interviewverfahren (K-SADS) eingesetzt: 65\% von 40 Kindern drogenabhängiger Mütter im Grundschulalter zeigten erhebliche Schwierigkeiten, den Schulanforderungen nachzukommen. Zu den Problemen gehörten Unaufmerksamkeit, schlechte Selbstdisziplin und Mangel an Beteiligung im Unterricht. Anpassungsschwierigkeiten in der Schule bestätigten auch Sowder und Burt [40]. Ihnen fiel unter den 8- bis 18-Jährigen eine erhöhte Neigung zum Schulschwänzen und zu schulischem Fehlverhalten auf, außerdem wurden die Kinder als aggressiver eingestuft, mit einer erhöhten Rate für Delinquenz.

Zusätzlich zur erhöhten Delinquenz wurden in neueren Untersuchungen [71,72] auffällig hohe Raten von Alkoholmissbrauch/-abhängigkeit (20\%), Nikotinabusus und Drogenabusus gefunden. 64\% der Nachuntersuchten hatten mit 11 Jahren bereits erste Delikte begangen, 21\% betrieben bereits Drogenabusus [72].

\section{Fremdunterbringung als Ausweg?}

In Anbetracht dieser Ergebnisse stellt sich die Frage, ob ein Verbleib des Kindes bei der Mutter überhaupt sinnvoll ist. Während bis zum Alter von einem Jahr 50-90\%, im Durchschnitt $73 \%$, der Kinder bei der Mutter aufwachsen, sind es nach dem ersten Lebensjahr nur noch 25-64\%. Aus eigenen Untersuchungen [44] geht hervor, dass von 101 Neugeborenen der Universitätsklinik Frankfurt $71 \%$ zu ihren Müttern entlassen wurden, 15\% kamen zu Pflegeeltern, 7\% zu Adoptiveltern und weitere $7 \%$ in ein Kinderheim. In einer dänischen Nachuntersuchung lebten nur noch 54\% der Kinder zwischen 1 und 10 Jahren bei ihrer drogenabhängigen Mutter. Sie wechselten durchschnittlich sechsmal das Milieu und fünfmal die Bezugsperson. Nur 25\% der Kinder konnten als unauffällig eingestuft werden, 56\% waren hyperaktiv, aggressiv und unkonzentriert [10]. Dieser hohe Prozentsatz ist mit großer Wahrscheinlichkeit auf den häufigen Wechsel der Bezugsperson zurückzuführen. Diese Beobachtungen werden von zwei Studien aus den Niederlanden bestätigt $[7,73]$, die schlechtere Entwicklungschancen für Kinder in Pflegefamilien als für Kinder mit Verbleib bei der Mutter verzeichneten. Ornoy et al. [74] fanden in ihrer Studie hingegen keine Unterschiede zwischen Kontrollgruppe und Pflegekindern, während die Kinder, die bei ihren opiatabhängigen Müttern aufwuchsen, Entwicklungsverzögerungen und vermehrt Verhaltensauffälligkeiten zeigten.

Aus eigenen Erfahrungen [44] ist hinzuzufügen, dass immer mehr Kinder mit HIV-Antikörpern geboren werden. Obwohl bis zum Alter von ungefähr einem Jahr noch nicht eindeutig festgestellt werden kann, ob diese Antikörper von der Mutter stammen oder ob das Neugeborene selbst HIV-positiv ist, sind diese Kinder kaum in eine Adoption zu vermitteln. Für die Hepatitis-Serologie gilt Ähnliches.

\section{Therapeutische Ansätze}

Seit 1999 sieht die Kassenärztliche Bundesvereinigung [75] in den Richtlinien über die Einführung neuer Untersuchungsund Behandlungsmethoden (NUB-Richtlinien) eine Indikation zur Substitutionsbehandlung bei i.v. Heroinabhängigen in der Schwangerschaft und bis sechs Monate nach der Geburt vor. Dies stellte einen längst überfälligen Schritt dar, da durch die lange Halbwertszeit des Methadons die für den Fetus gefährlichen starken Schwankungen des Opiatspiegels vermieden werden können. Außerdem zeigte sich in unserer Untersuchung an der Frankfurter Universitätsklinik [76] deutlich, dass die methadonsubstituierten Mütter in stabileren sozioökonomischen Verhältnissen lebten als nicht substituierte Frauen und sich signifikant besser um ihre Kinder kümmerten: $81,3 \%$ der substituierten Frauen besuchten ihre Kinder regelmäßig in der Neonatologie, das Zuwendungsverhalten war bei $90,9 \%$ angemessen. Allerdings hatten, wie bereits aus früheren Untersuchungen bekannt $[53,54]$, die Neugeborenen unter Methadon einen erheblich schwereren Entzug mit häufigeren Krampfanfällen.

Eine Alternative bietet möglicherweise die Substitution mit Buprenorphin (in Deutschland: Temgesic ${ }^{\circledR}$ ), wie sie von einer Arbeitsgruppe der Universitätsklinik in Wien bereits seit einigen Jahren angewandt wird [77; Ortner et al. in diesem Heft]; in einer aktuellen Publikation [78] beschreiben die Autoren Befunde von 15 Schwangeren und Neugeborenen unter Substitution mit 1-10 mg Buprenorphin/Tag mit guter Verträglichkeit und milden bis mäßigen Entzugssymptomen des Neugeborenen.

Schwangere Opiatabhängige benötigen ab dem frühestmöglichen Zeitpunkt eine umfassende interdisziplinäre Betreuung. Ab 1987 wurde beispielsweise am Maudsley Hospital London ein spezieller Liaison-Service eingerichtet [47]. Das erste deutsche Projekt „IGLU“ für drogenabhängige Mütter und ihre Kinder begann 1989 in Hamburg und wurde durch den Trägerverein „Palette e. V.“ initiiert mit dem Schwerpunkt Beratung und stützende soziale Hilfen [79]. 
Evaluationen psychosozialer Betreuungsprogramme für drogenabhängige Eltern und deren Kinder in Form aufsuchender Interventionen zu Hause $[80,81]$ oder stationärer Familienprogramme [82] i. S. von Kompetenztraining konnten positive Effekte auf die Erziehungskompetenz der drogenabhängigen Eltern nachweisen.

Der 104. Deutsche Ärztetag forderte im Mai 2001 in einer Entschließung ein Case-Management für Kinder von Drogenabhängigen und deren Eltern. Hierzu wurde gefordert, „dass Suchtmediziner, Hausärzte, Gynäkologen, Pädiater und Kinder- und Jugendpsychiater entsprechende Kooperationsvereinbarungen mit ihren Jugendämtern treffen“ ... „Die anderweitige ,Fremdunterbringung dieser Kinder kann so vielfach verhindert werden“ [83].

\section{Methodenkritik}

Bei der Suche nach relevanter Literatur zu diesem Thema hat sich herausgestellt, dass es nur wenige Studien gibt, die eine Kontrollgruppe zum Vergleich heranziehen. Die wenigen existierenden Fall-Kontroll-Studien vergleichen Gruppen opiatabhängiger Mütter mit drogenfreien Kontrollgruppen. Dabei werden aber nicht immer Parallelisierungskriterien wie Alter der Mutter, Parität und soziale Schicht beachtet [84].

Die Abschätzung der Effektstärke der verschiedenen biologischen und psychosozialen Einflussgrößen auf die Entwicklung der Kinder bringt erhebliche methodische Probleme mit sich; so ist die Rolle des Konsums einer bestimmten Droge (z.B. Kokain, siehe [85]) während der Schwangerschaft hinsichtlich der Entwicklung des Kindes kaum abschätzbar, da eine Vielzahl konfundierender Variablen (schwer einzuschätzender Beigebrauch anderer Substanzen, wie Cannabis [86-88], sehr hoher Nikotin- [89] und Alkoholkonsum [90,91], spezielle psychosoziale Risiken) hinzukommt. Diese kovariierenden Faktoren sind selbst sehr starke Risikofaktoren für negative Entwicklungen, daher gehört die Kontrolle der konfundierenden Einflüsse zu den wichtigsten methodischen Problemen in diesem Feld [92]. Suchman und Luthar [93] verglichen in einer methodisch sehr aufwändigen Untersuchung mütterliches Erziehungsverhalten zwischen opiatabhängigen und nicht abhängigen Müttern, die sorgfältig nach sozioökonomischem Status parallelisiert wurden; dabei zeigte sich, dass ausschließlich fehlende mütterliche Anteilnahme und Initiative auf die Abhängigkeitserkrankung zurückgeführt werden können, alle anderen Unterschiede im Erziehungsverhalten jedoch durch sozioökonomische bzw. psychosoziale Variablen erklärt werden können.

\section{Literatur}

${ }^{1}$ Hogan DM. Annotation: the psychological development and welfare of children of opiate and cocaine users: review and research needs. Journal of Child Psychology and Psychiatry and Allied Disciplines 1998; 39: 609-620

2 Perkonigg A, Lieb R, Wittchen HU. Prevalence of use, abuse and dependence of illicit drugs among adolescents and young adults in a community sample. Eur Addict Res 1998; 4: 58-66

${ }^{3}$ Langstein L. Über das Schicksal von morphinsüchtigen Frauen geborene Säuglinge. Medizinische Klinik 1930; 14: 500-501

${ }^{4}$ Weiss RF. Über die Behandlung der Neugeborenen morphiumsüchtiger Frauen. Medizinische Klinik 1930; 32
${ }^{5}$ Berridge V, Edwards G. Opium and the People. New Haven and London: Yale University Press, 1987

${ }^{6}$ Marcus J, Hans SL, Jeremy RJ. Patterns of 1-day and 4-month motor functioning in infants of women on methadone. Neurobehavioral Toxicol Teratol 1982; 4: 473-476

${ }^{7}$ Groeneweg B, van de Lechner-Noort M. Kinderen van drugverslaafde ouders, opvoeding en ontwikkeling. Delft: Eburon, 1988

${ }^{8}$ Little BB, Snell LM, Knoll KA et al. Heroin abuse during pregnancy: effects on perinatal outcome and early childhood growth. Am J Hum Biol 1991; 3: 463-468

${ }^{9}$ Hutchings DE. Opiates during pregnancy: neurobehavioral effects in the offspring. In: Nahas GG, Latour C, Hardy N, Dingeon P (Hrsg). Physiopathology of illicit drugs. Oxford: Pergamon, 1991: 285-296

${ }^{10}$ Olofsson M, Buckley W, Andersen GE, Friis-Hansen B. Investigation of 89 children born by drug-dependent mothers: neonatal course. Acta Paediatr Scand 1983; 72: 403-406

${ }^{11}$ Bundesministerium für Gesundheit. Pressemitteilung 1997; Nr. 13, 17. Feb. 1997

${ }^{12}$ Hessisches Ärzteblatt. Drogensüchtige Neugeborene leiden qualvollen Entzug. Hessisches Ärzteblatt 1994; 8: 238

${ }^{13}$ Kleiber D, Pant A. HIV-Needle-Sharing-Sex. Eine sozialepidemiologische Studie zur Analyse der HIV-Prävalenz und riskanter Verhaltensweisen bei i.v.-Drogenkonsumenten. Schriftenreihe des Bundesministeriums für Gesundheit, Baden-Baden: Nomos, 1996; Bd. 69a

14 Türk D, Welsch K. EBIS-Jahresstatistik 1999 der ambulanten Beratungs- und Behandlungsstellen für Suchtkranke in Deutschland. Sucht 2000; 46: S7-S52

${ }^{15}$ Bucher HU, Dahlem P, Cuendet D. Prävalenz von Drogen im Mekonium. Schweizer Med Wochenschrift 1991; 101: 6

${ }^{16}$ Wolstein J, Wirth I, Wolstein R, Gastpar M. Methadonsubstitution in der Schwangerschaft. Sucht 1999; 45: 306-314

17 Davis SK. Chemical dependency in women: A description of it's effects and outcome on adequate parenting. J Subst Abuse Treat 1990; 7: 225-232

${ }^{18}$ Coyer SM. Mothers recovering from cocaine addiction: Factors affecting parenting skills. J Obstet Gynecol Neonatol Nurs 2001; 30: 71-79

${ }^{19}$ Stauber M, Schwerdt B, Hollenbach B. Schwangerschaft, Geburt und Wochenbett bei heroinabhängigen Frauen - derzeitiger Wissensstand und eigene Erfahrungen. Geburtshilfe Frauenheilkd 1982; 42: 345-352

${ }^{20}$ Jarvis TJ, Copeland J, Walton L. Exploring the nature of the relationship between child sexual abuse and substance use among women. Addiction 1998; 93: 865-875

${ }^{21}$ Stauber M, Weingart-Jesse B. Psychosomatische Aspekte der Suchterkrankung während Schwangerschaft, Geburt und Wochenbett unter Berücksichtigung des weiteren Verlaufes. Gynäkologe 1991; 24: 345-348

${ }^{22}$ Harmer AL, Sanderson J, Mertin P. Influence of negative childhood experiences on psychological functioning, social support, and parenting for mothers recovering from addiction. Child Abuse and Neglect 1999; 23: 421-433

${ }^{23}$ Schafer M, Schnack B, Soyka M. Sexueller und körperlicher Missbrauch während früher Kindheit oder Adoleszenz bei späterer Drogenabhängigkeit. Psychotherapie, Psychosomatik, Medizinische Psychologie 2000; 50: 38-50

${ }^{24}$ Marcenko MO, Kemp SP, Larson NC. Childhood experiences of abuse, later substance use, and parenting outcomes among low-income mothers. American Journal of Orthopsychiatry 2000; 70: 316-326

${ }^{25}$ Salloum IM, Mezzich JE, Cornelius JR, Daley DC. Comorbidity in drug abuse: The U. S. experience. In: Beigel A, Lopez Ibor JJ, Costa e Silva JA (Hrsg). Past, Present and Future of Psychiatry. IX. World Congress of Psychiatry. Singapore: World Scientific, 1994 
${ }^{26}$ Marsden J, Gossop M, Stewart D, Rolfe A, Farrell M. Psychiatric symptoms among clients seeking treatment for drug dependence. Brit J Psychiat 2000; 176: 285-289

${ }^{27}$ Kuntze MF, Ladewig D, Stohler R. Art und Häufigkeit der Komorbidität bei methadonsubstituierten Opiatabhängigen in der ambulanten Versorgung. Sucht 1998; 44: 95-103

${ }^{28}$ Günthner A, Dednder C, Schäfer G et al. Komorbidität bei Drogenabhängigen. Empirische Ergebnisse und therapeutische Konsequenzen. Suchttherapie 2000; 1: 16-20

${ }^{29}$ Black R, Mayer J. Parents with special problems: alcoholism and opiate addiction. Child Abuse Negl 1980; 4: 45-54

${ }^{30}$ Burns K, Melamed J, Burns W et al. Chemical dependence and clinical depression in pregnancy. J Clin Psychol 1985; 41: 851-854

${ }^{31}$ Freier K. In utero exposure and maternal-infant interaction: the complexities of the dyad and their environment. Infant Mental Health J 1994; 15: 176-188

32 Bernstein V, Jeremy RJ, Hans S, Marcus J. A longitudinal study of offspring born to methadone-maintained women. II. Dyadic interaction and infant behavior at four months. Am J Drug Alcohol Abuse 1984; 10: 161

${ }^{33}$ van Baar AL. The Development of Infants of Drug Dependent Mothers. Amsterdam: Swets \& Zeitlinger, 1991

${ }^{34}$ Bauman P, Dougherty F. Drug-addicted mothers' parenting and their children's development. Int J Addict 1983; 18: 291-302

35 Bays J. Substance abuse and child abuse. Impact of addiction on the child. Pediatr Clin North Amer 1990; 37: 881-904

${ }^{36}$ Gossop M, Griffiths P, Strang J. Sex differences in patterns of drug taking behavior. A study at a London community drug team. Brit J Psychiat 1994; 164: 101-104

${ }^{37}$ Fraser AC, Cavanagh S. Pregnancy and drug addiction - longterm consequences. J R Soc Med 1991; 84: 530-532

${ }^{38}$ Kelly JJ, Davis PG, Henschke PN. The drug epidemic: effects on newborn infants and health resource consumption at a tertiary perinatal centre. Journal of Paediatrics and Child Health 2000; 36: $262-264$

${ }^{39}$ Randall $\mathrm{T}$. Intensive prenatal care may deliver healthy babies to pregnant drug abusers. JAMA 1991; 265: 2773-2774

40 Sowder BJ, Burt MR. Children of Heroin Addicts: An assessment of health, learning, behavioral and adjustment problems. New York: Praeger, 1980

${ }^{41}$ Lejeune C, Ropert JC, Montamat S et al. Devenir médico-social de 59 nouveau-nés de mère toxicomane. J Gynecol Obstet Biol Reprod (Paris) 1997; 26: 395-404

42 Casado-Flores J, Bano-Rodrigo A, Romer E. Social and medical problems in children of heroin-addicted parents. Am J Dis Child 1990; 144: 977-979

${ }^{43}$ Rosen TS, Johnson HL. Children of methadone-maintained mothers: follow-up to 18 months of age. J Pediatr 1982; 101: 192-196

${ }^{44}$ Ziegler M. Medizinische und psychosoziale Risikofaktoren für spätere kinder- und jugendpsychiatrische Auffälligkeiten bei Kindern opiatabhängiger Mütter. Dissertation Fachbereich $\mathrm{Hu}-$ manmedizin der J.W. Goethe-Universität Frankfurt am Main, 1998

${ }^{45}$ Blanche S, Rouzioux C, Guihard Moscato ML et al. A prospective study of infants born to women seropostive for human immunodeficiency virus type 1. N Engl J Med 1989; 320: 1643-1648

${ }^{46}$ European Collaborative Study. Mother-to-child transmission of HIV infection. Lancet 1988; II (8619): 1039-1042

${ }^{47}$ Dawe S, Gerada C, Strang J. Establishment of a liason service for pregnant opiate-dependent women. $\mathrm{Br} \mathrm{J}$ Addict 1992; 87: $867-871$

${ }^{48}$ Bode $\mathrm{H}$, Fabian T, Rudin $\mathrm{C}$ et al. Kinder drogenabhängiger Mütter. Klin Pädiatr 1992; 204: 92-97
${ }^{49}$ Vering A, Seeger J, Becker S et al. Heroinabusus und MethadonSubstitution in der Schwangerschaft. Geburtshilfe Frauenheilkd 1992; 52: 144-147

${ }^{50}$ Finnegan LP. Neonatal abstinence. In: Nelson NM (Hrsg). Current Therapy in Neonatal-Perinatal Medicine (2. Aufl.). Toronto: Decker, 1985: 262-270

${ }^{51}$ Agarwal P, Rajadurai VS, Bhavani S, Tan KW. Perinatal drug abuse in KK Women's and Children's Hospital. Annals of the Academy of Medicine, Singapore 1999; 28: 795-799

${ }^{52}$ Hutchings DE, Zmitrovich AC. Methadone during pregnancy: A brief review of clinical outcome and a new animal model. In: Lewis M, Bendersky M (Hrsg). Mothers, Babies, and Cocaine: The Role of Toxins in Development. Hillsdale: Lawrence Erlbaum Assoc, 1995

${ }^{53}$ Stimmel B, Adamsons K. Narcotic dependency in pregnancy. JAMA 1976; 235: 1121-1124

${ }^{54}$ Rajegowda BF, Glass L, Evans HE et al. Methadone withdrawal in newborn infants. J Pediatr 1972; 81: 532-534

${ }^{55}$ Finnegan LP, Kandall SR. Neonatal Abstinence. In: Lowinson JH, Ruiz P, Millman RB (Hrsg). Substance Abuse: A Comprehensive Textbook. (2. Aufl.). Baltimore: Williams \& Wilkins, 1992

${ }^{56}$ Kaltenbach K, Finnegan LP. Perinatal and developmental outcome of infants exposed to methadone in utero. Neurotoxicol Teratol 1987; 9: 311-313

${ }^{57}$ Kaltenbach K, Finnegan LP. Developmental outcome of infants exposed to methadone in utero. Pediatr Res Abst 1986; 20: 57A

58 Doberczak TM, Shanzer S, Cutler R et al. One-year follow-up of infants with abstinence-associated seizures. Arch Neurol 1988; 45: 649-653

${ }^{59}$ Lifschitz MH, Wilson GS, Smith EO, Desmond MM. Factors affecting head growth and intellectual function in children of drug addicts. Pediatrics 1985; 75: 269-274

${ }^{60}$ van Baar AL, Soepatmi S, Gunning WB, Akkerhuis GW. Development after prenatal exposure to cocaine, heroin and methadone. Acta Paediatr Scand 1994; 83: 40-46

${ }^{61}$ Sardeman H, Madsen KS, Friis-Hansen B. Follow-up of children of drug-addicted mothers. Arch Dis Childh 1976; 51: 131-134

62 Wilson GS, McCreary R, Kean J, Baxter JC. The development of preschool children of heroin addicted mothers: a controlled study. Pediatrics 1979; 63: 135-141

${ }^{63}$ van Baar AL. Development of infants of drug dependent mothers. J Chil Psychol Psychiat 1990; 31: 911-920

${ }^{64}$ Koren G, Nulman I, Rovet J et al. Long-term neurodevelopmental risks in children exposed in utero to cocaine. The Toronto Adoption Study. Annals of the New York Academy of Sciences 1998; 846: 306-313

65 de Cubas MM, Field T. Children of methadone-dependent women: Developmental outcomes. Am J Orthopsychiatry 1993; 63: 266-276

${ }^{66}$ Griffith DR, Azuma SD, Chasnoff IJ. Three-year outcome of children exposed prenatally to drugs. J Am Acad Child Adolesc Psychiatry 1994; 33: 20-27

${ }^{67}$ Wilens TE, Biederman J, Kiely K et al. Pilot study of behavioral and emotional disturbances in the high-risk children of parents with opioid dependence. J Am Acad Child Adolesc Psychiatry 1995; 34: 779-785

${ }^{68}$ Chasnoff IJ, Anson A, Hatcher R et al. Prenatal exposure to cocaine and other drugs. Outcome at four to six years. Annals of the New York Academy of Sciences 1998; 846: 314-328

${ }^{69}$ Achenbach TM. Manual for the Child Behavior Checklist/4-18 and 1991 Profile. Burlington: University of Vermont, Dept. of Psychiatry, 1991

${ }^{70}$ Wilson GS. Clinical studies of infants and children exposed prenatally to heroin. Ann NY Acad Sci 1989; 562: 183-194 
${ }^{71}$ Merikangas KR, Dierker LC, Szatmari P. Psychopathology among offspring of parents with substance abuse and/or anxiety disorders: a high-risk study. Journal of Child Psychology and Psychiatry and Allied Disciplines 1998; 39: 711-720

72 Nurco DN, Blatchley RJ, Hanlon TE, O’Grady KE. Early deviance and related risk factors in the children of narcotic addicts. American Journal of Drug and Alcohol Abuse 1999; 25: 25-45

${ }^{73}$ Soepatmi S. Developmental outcomes of children of mothers dependent on heroin or heroin/methadone during pregnancy. Acta Paediatr Scand 1994; 83: 36-39

${ }^{74}$ Ornoy A, Michailevskaya V, Lukashov I et al. The developmental outcomes of children born to heroin-dependent mothers, raised at home or adopted. Child Abuse Negl 1996; 20: 385-396

${ }^{75}$ Kassenärztliche Bundesvereinigung. Richtlinien des Bundesausschusses der Ärzte und Krankenkassen über die Einführung neuer Untersuchungs- und Behandlungsmethoden (geänderte Fassung von 26.4.1999). Deutsches Ärzteblatt 1999; 96: B 1382-1384

${ }^{76}$ Ziegler M, Poustka F, von Loewenich V, Englert E. Postpartale Risikofaktoren in der Entwicklung von Kindern opiatabhängiger Mütter: Ein Vergleich zwischen Müttern mit und ohne Methadon-Substitution. Nervenarzt 2000; 71: 730-736

77 Schneider C, Fischer G, Diamant K et al. Pregnancy and drug dependence. Wien Klin Wochenschr 1996; 108: 611-614

78 Fischer G, Johnson RE, Eder H et al. Treatment of opioid-dependent pregnant women with buprenorphine. Addiction 2000; 95 : 239-244

79 von Soer J, Strathenwerth I. Süchtig geboren. Kinder von Heroinabhängigen. Hamburg: Rasch und Röhring, 1991

${ }^{80}$ Catalano RF, Gainey RR, Fleming CB et al. An experimental intervention with families of substance abusers: one-year follow-up of the focus on families project. Addiction 1999; 94: 241-254

${ }^{81}$ Schuler ME, Nair P, Black MM, Kettinger L. Mother-infant interaction: Effects of a home intervention and ongoing maternal drug use. J Clin Child Psychol 2000; 29: 424-431

82 Keen J, Oliver P, Rowse G, Mathers N. Keeping families of heroin addicts together: results of 13 months' intake for community detoxification and rehabilitation at a family centre for drug users. Family Practice 2000; 17: 484-489

83 Deutsches Ärzteblatt. Dokumentation 104. Deutscher Ärztetag vom 22. bis 25. Mai 2001. Entschließungen zum Tagesordnungspunkt V. Deutsches Ärzteblatt 2001; 98: B 1264

${ }^{84}$ Carta J, Sideridis G, Rinkel P et al. Behavioral outcomes of young children prenatally exposed to illicit drugs: review and analysis of experimental literature. Top Early Child Spec Educ 1994; 14: 184-216

${ }^{85}$ Richardson GA, Day NL. Alcohol, marijuana, and tobacco: effects of prenatal exposure on offspring growth and morphology at age six. Alcohol Clin Exp Res 1994; 18: 786-794

${ }^{86}$ Frank D, Bauchner H, Parker S et al. Neonatal body proportionality and body composition after in utero exposure to cocaine and marijuana. J Pediatr 1990; 117: 622-626

${ }^{87}$ Zuckerman B, Frank D, Hingson R et al. Effects of maternal marijuana and cocaine use on fetal growth. N Engl J Med 1989; 320: 762-768

${ }^{88}$ Fried PA, Buckingham M, von Kulmitz P. Marijuana use during pregnancy and perinatal risk factor. Am J Obste Gynecol 1983; 144: 922

${ }^{89}$ McDonald AD, Armstrong BG, Sloan M. Cigarette, alcohol, and coffee consumption and prematurity. Am J Public Health 1992; 82: 87-90

${ }^{90}$ Streissguth AP, Barr HM, Sampson PD et al. Neurobehavioral effects of prenatal alcohol. Part 1: Literature review and research strategy. Neurotoxicol Teratol 1989; 11: 461-476
${ }^{91}$ Larsson G, Bohlin AB, Tunell R. Prospective study of children exposed to variable amounts of alcohol in utero. Arch Dis Child 1985; 60: 316-321

92 Jacobson JL, Jacobson SW. Strategies for detecting the effects of prenatal drug exposure: Lessons from research on alcohol. In: Lewis M, Bendersky M (Hrsg). Mothers, Babies, and Cocaine: The Role of Toxins in Development. Hillsdale: Lawrence Erlbaum Assoc, 1995

${ }^{93}$ Suchman NE, Luthar SS. Maternal addiction, child maladjustment and socio-demographic risks: implications for parenting behaviors. Addiction 2000; 95: 1417-1428

${ }^{94}$ Klenka HM. Babies born in a district general hospital to mothers taking heroin. BMJ 1986; 293: 745-746

${ }^{95}$ van Baar AL, Fleury P, Soepatmi S et al. Neonatal behaviour after drug dependent pregnancy. Arch Dis Child 1989; 64: 235-240

${ }^{96}$ Fanshel D. Parental failure and consequences for children: The drug abusing mother whose children are in foster care. Amer J Publ Health 1975; 65: 604-612

${ }^{97}$ Strauss ME, Starr RH, Ostrea EM jr et al. Behavioral concomitants of prenatal addiction to narcotics. J Pediatr 1976; 89: 842-846

${ }^{98}$ Bauman P, Levine SA. The development of children of drug addicts. Int J Addict 1986; 21: 849-863

\section{Dr. med. Ekkehart D. Englert}

Klinik für Kinder- und Jugendpsychiatrie und Psychotherapie HELIOS Klinikum Erfurt

Nordhäuser Straße 74

99089 Erfurt

E-mail: EEnglert@erfurt.helios-kliniken.de 